Research Paper

\title{
Investigation of metastasis-associated in colon cancer-1 genetic variants in the development and clinicopathologcial characteristics of uterine cervical cancer in Taiwanese women
}

\author{
Yi-Hung Sun 1,2 , Ying-Hsiang Chou 1,3,4, Chu-Chyn Ou,5,6, Soo-Cheen Ng7,8, Huang-Pin Shen 1,7,8, Yueh-Chun \\ Lee $^{1,4,7}$, Chun-Fang Hsu ${ }^{1}$, Shun-Fa Yang ${ }^{1,6}$, Po-Hui Wang ${ }^{1,6,7,8}$ \\ 1. Institute of Medicine, Chung Shan Medical University, Taichung, Taiwan. \\ 2. Department of Obstetrics and Gynecology, Chi-Mei Foundation Medical Center, Tainan, Taiwan. \\ 3. Department of Medical Imaging and Radiological Sciences, Chung Shan Medical University, Taichung, Taiwan. \\ 4. Department of Radiation Oncology, Chung Shan Medical University Hospital, Taichung, Taiwan. \\ 5. School of Nutrition, Chung Shan Medical University, Taichung, Taiwan. \\ 6. Department of Medical Research, Chung Shan Medical University Hospital, Taichung, Taiwan. \\ 7. School of Medicine, Chung Shan Medical University, Taichung, Taiwan. \\ 8. Department of Obstetrics and Gynecology, Chung Shan Medical University Hospital, Taichung, Taiwan. \\ $\square$ Corresponding author: Po-Hui Wang, Institute of Medicine, Chung Shan Medical University, 110, Section 1, Chien-Kuo North Road, Taichung, 40201, \\ Taiwan. Tel.: 886-4-24739595 ext. 21721; Fax: 884-4-24738493; E-mail: ginhow84921344@yahoo.com.tw
}

() The author(s). This is an open access article distributed under the terms of the Creative Commons Attribution License (https://creativecommons.org/licenses/by/4.0/). See http://ivyspring.com/terms for full terms and conditions.

Received: 2019.09.11; Accepted: 2019.12.23; Published: 2020.02.04

\begin{abstract}
The objectives of this study were to define the associations among single nucleotide polymorphisms (SNPs) of metastasis-associated in colon cancer-1 (MACCI) gene, development and clinicopathological characteristics of uterine cervical cancer, and patient survival in Taiwan. Genotypic frequencies of 5 MACCI SNPs rs975263, rs3095007, rs4721888, rs3735615 and rs1990172 were identified for 132 patients with invasive cancer, 99 with high-grade cervical intraepithelial neoplasia and 338 normal controls using real-time polymerase chain reaction. It revealed that there were no associations of these MACCI SNPs with cervical carcinogenesis. In the meantime, cervical cancer patients with genotype GG in MACCI SNP rs975263 tended to display more risk to have vaginal invasion than those with AA/AG ( $p=0.042$, OR: 8.70, 95\% Cl: 0.81-433.22). In multivariate analysis, positive pelvic lymph node metastasis could significantly predict worse 5 years survival rate $(p=0.001 ; \mathrm{HR}=9.98,95 \% \mathrm{Cl}=2.64-37.77)$ for cervical cancer patients. In conclusion, pelvic lymph node status rather than MACCI SNPs was the only independent parameter that could significantly predict 5 years survival rate in Taiwanese women with cervical cancer.
\end{abstract}

Key words: metastasis-associated in colon cancer-1; single nucleotide polymorphisms; uterine cervical cancer; vaginal invasion; 5 years survival rate

\section{Introduction}

Metastasis-associated in colon cancer-1 (MACC1) gene, which is related to colon cancer, was in the beginning found by Stein et al., using genome-wide expression method for an unique gene that was differently expressed in human primary colon cancer and metastatic tissues, as well as normal tissues [1]. The MACC1 gene is situated on chromosome 7 at position $7 \mathrm{p} 21.1$ and encodes the hepatocyte growth factor (HGF) receptor as well as MET, and then modulates HGF-MET signaling pathway [1]. Elevated MACC1 expression has been demonstrated to be associated with tumor oncogenesis, metastasis and worse prognosis, as well as regarded as an early risk factor for cancer patients [1-6]. In addition, it has been revealed that elevated MACC1 is correlated with cancer tissues of uterine 
cervix, while compared with normal cervical tissues [7]. Its high expression was also found to be associated with aggressive phenotypes of cervical cancer.

Uterine cervical cancer is the fourth most common cancer in women worldwide [8]. Taiwan 2013 annual cancer registry report revealed that it was the seventh most common cancer in this country. Cervical intraepithelial neoplasias (CINs) are considered as the precursor lesions of cervical cancer [9]. Cervical carcinogenesis is a multistep progression and is exhibited as a continued process of neoplastic transition from CIN to invasive cancer of uterine cervix $[10,11]$. CINs are histologically subdivided into CIN 1 (mitoses and immature cells in the lower third of the cervical epithelium; low-grade CIN) as well as CIN 2 and CIN 3 (mitoses and immature cells separately in the middle and upper third of the cervical epithelium; high-grade CIN) with progressive severity.

If the shared sequence of a gene exhibits a different single nucleotide between the individuals of a species, or paired chromosomes in an individual with a frequency of more than $5 \%$, single nucleotide polymorphism (SNP) is defined [12]. These genetic variants may have an impact on the promoter activity and gene expression. The relationships of the MACC1 SNPs with clinical variables of colon cancer have ever been demonstrated [13]. However, the impact of MACC1 SNPs on the development and clinical outcome of cervical cancer has not been explored yet. Therefore, we conducted this study to investigate the involvement of the following 5 MACC1 SNPs rs975263, rs3095007, rs4721888, rs3735615 and rs1990172 in the development and clinicopathological characteristics of cervical cancer and patient prognosis in Taiwanese women.

\section{Materials and Methods}

\section{Population}

This retrospective study was designed by consecutively recruiting one hundred and thirty-two patients with invasive cancer and 99 women with high-grade CIN of uterine cervix from the Department of Obstetrics and Gynecology in Chung Shan Medical University Hospital in Taichung, Taiwan from February 1994 to October 2014. Meanwhile, 338 normal women, who received general examination at the Outpatient Patient Department in this hospital, were recruited as controls. The diagnosis for patients with invasive cervical cancer and those with high-grade CIN were included based on pathological report from colposcopy-directed cervical biopsy. Thereafter, cervical cancer patients underwent the standard treatment protocols, revised from guidelines provided by National Comprehensive Cancer Network and those with high-grade CIN, who were known to have precancerous lesions, underwent large loop excision of transformation zone, simple trachelectomy, abdominal or vaginal total hysterectomy. The normal controls were discriminated based on the cytologic report from cervical Papanicolaou smear and the report was further clarified by normal colposcopic findings. All subjects were Taiwanese women residing in central Taiwan. The study was approved by the Chung Shan Medical University Hospital institutional review board (CSMUH No: CS18208).

\section{Deoxyribonucleic acid (DNA) extraction from blood samples in all individuals and selection of MACCI SNPs}

The laboratory staff drew the blood samples from all participants using venipuncture technique. The specimens were collected into Vacutainer tubes mixed with ethylenediaminetetraacetic acid. They were stored at $4^{\circ} \mathrm{C}$ shortly. DNA was extracted from leukocytes according to previous publication [14]. The extracted DNA was then dissolved into $\mathrm{pH}$ 7.8 TE buffer. Hereafter, it was quantified by the measurement of OD260. The OD260/OD280 ratio was checked and the range of 1.8-2.0 conformed to our criteria and defined as pure to prevent its cross reactivity from the present homologous RNA in the samples. The final products were then stored at $-20^{\circ} \mathrm{C}$ and were used as templates for the polymerase chain reaction (PCR).

Five MACC1 genetic polymorphisms were selected based on the data of International HapMap Project and previous work [13]. The five MACC1 SNPs were selected because these SNPs were suggested to be associated with the risk of cancer susceptibility [15-18]. MACC1 SNPs rs975263, rs3095007, rs4721888, rs3735615 and rs1990172 were checked by ABI StepOne Real-Time PCR System (Applied Biosystems, Foster City, CA, USA), and determined with SDS vers. 3.0 software, as our previous publication [19].

\section{Statistical analysis}

Analysis of variance (ANOVA) was applied for the comparison of the age distribution in the studied individuals using Tukey HSD test for post hoc analysis. Hardy-Weinberg equilibrium was performed to assess the genotypic frequencies of rs975263, rs3095007, rs4721888, rs3735615 and rs1990172 in normal controls [degree of freedom (d.f. $=2]$. Chi-square or Fisher exact tests were applied to explore the relationships of a variety of MACC1 
genetic distributions with the cervical carcinogenesis. The odds ratio (OR), adjusted odds ratio (AOR) and their 95\% confidence intervals (95\% CIs) were calculated using logistic regression model or multinomial logistic regression model after age adjustment. These tests were also performed to relate MACC1 SNP frequencies with various clinicopathological parameters.

Kaplan-Meier curves were applied to plot the impacts of MACC1 SNPs and clinicopathological characteristics for 5 years survival in univariate analysis. The log-rank test was performed to identify the differences between these curves. Cox proportional hazard model was performed to evaluate the impacts of MACC1 SNPs and various clinicopathological parameters on 5 years survival in multivariate analysis relative to survival time. The SPSS, version 12.0 and WinPepi Software, version 10.0 were applied for statistical analysis. Hazard ratios (HRs) and their 95\% confidence intervals (CI) were defined by the SPSS, version 12.0. $P<0.05$ was regarded as statistically significant difference.

\section{Results}

\section{Age distribution}

There was a statistical difference for age distribution between patients with cervical neoplasia and normal control women ( $50.9 \pm 13.6$ vs. $43.9 \pm 10.4$, $p<0.001)$. Using ANOVA with Tukey HSD test for post hoc analysis, the age difference was statistically significant between patients with cervical invasive cancer and those with precancerous lesion (55.4 \pm 12.2 vs. $44.9 \pm 13.0, p<0.001$ ) as well as between those with cervical cancer and control women $(55.4 \pm 12.2$ vs. 43.9 $\pm 10.4, p<0.001$ ) but not statistically significant between women with precancerous lesions and control women $(44.9 \pm 13.0$ vs. $43.9 \pm 10.4, p=0.730)$.

\section{Hardy-Weinberg equilibrium}

The minor allele frequencies of MACC1 SNPs rs975263, rs3095007, rs4721888, rs3735615 and rs1990172 in control women were all $\geq 5 \%$. In control women, genotypic frequency of MACC1 SNP rs 975263 conformed to the Hardy-Weinberg equilibrium [ $x^{2}$ value, 2.008, $p=0.366$; d.f.=2]. The frequencies of MACC1 SNPs s3095007, rs4721888, rs3735615 and rs1990172 were also satisfied the Hardy-Weinberg equilibrium ( $\chi^{2}$ value, 0.090; $\chi^{2}$ value, $0.210 ; \chi^{2}$ value, 0.598 and $\chi 2$ value, 0.507 , respectively).

\section{Association of $\mathrm{MACCl}$ genetic variants with cervical carcinogenesis}

There was no statistically different in the frequencies of MACC1 SNP rs975263 between the patients with cervical neoplasia and the normal controls ( $p=0.511$; Table 1). Even after adjusting for age, no significant difference still existed (Table 1). While using wild-type homogenous genotype AA as a reference, heterogeneous AG $(p=0.891$; AOR $=1.03$, $95 \% \mathrm{CI}=0.71-1.52)$ or variant homogenous genotype GG $(p=0.275$; AOR $=0.58,95 \% \mathrm{CI}=0.21-1.55)$ as well as AG/GG $(p=0.844 ;$ AOR $=0.96,95 \% \quad C I=0.66-1.40)$ could not exert statistically significant distributions after age adjustment between patients with cervical neoplasia and the normal controls (Table 1). While using $\mathrm{AA} / \mathrm{AG}$ as references, variant homogenous genotype GG also exhibited no statistical difference between them $(p=0.265$; AOR $=0.57,95 \% \mathrm{CI}=0.21-1.53$; Table 1). In the meantime, neither was statistical differences observed in other MACC1 SNPs between the women with cervical neoplasia and the normal controls (Table 1), nor did significant difference after controlling for age in these SNPs (Table 1).

When the patients with cervical neoplasia group was further classified into subgroups of those with invasive cancer or pre-cancerous lesions, no statistical differences were found in the frequencies of MACC1 SNP rs975263 among the patients with cervical invasive cancer and pre-cancerous lesions as well as the normal controls $(p=0.610$; Table 2$)$. There was no statistical difference, in the frequency comparison of heterozygote AG and variant homozygote GG and AG/GG of MACC1 SNP rs975263 using wild-type homozygote AA as a reference after age adjustment, between patients with cervical pre-cancerous lesions and normal controls $(p=0.917, \mathrm{AOR}=0.97,95 \%$ $\mathrm{CI}=0.59-1.61 ; p=0.141, \quad \mathrm{AOR}=0.22,95 \% \mathrm{CI}=0.03-1.67$ and $p=0.574, \quad \mathrm{AOR}=0.87, \quad 95 \% \quad \mathrm{CI}=0.53-1.42$, respectively) as well as between patients with cervical invasive cancer and normal controls $(p=0.810$, $\mathrm{AOR}=1.06,95 \% \mathrm{CI}=0.65-1.73 ; p=0.954, \mathrm{AOR}=0.97,95 \%$ $\mathrm{CI}=0.32-2.92$ and $p=0.843, \quad \mathrm{AOR}=1.05, \quad 95 \%$ $\mathrm{CI}=0.66-1.67$, respectively). Additionally, there was also no statistical difference in the frequencies of variant homogenous genotype GG of MACC1 SNP rs975263 using AA/AG as references after age adjustment between patients with cervical pre-cancerous lesions and normal controls $(p=0.143$, $\mathrm{AOR}=0.22,95 \% \mathrm{CI}=0.03-1.67)$ as well as between those with cervical invasive cancer and normal controls $(p=0.928, \mathrm{AOR}=0.95,95 \% \mathrm{CI}=0.32-2.84$; Table $2)$. There were also no statistically different frequencies of other MACC1 SNPs between patients with cervical pre-cancerous lesions and normal controls as well as between those with cervical invasive cancer and normal controls (Table 2). 
Table 1. Genetic polymorphism distributions of the metastasis-associated in colon cancer-1 gene in Taiwanese women with neoplasias of the uterine cervix and normal controls

\begin{tabular}{|c|c|c|c|c|c|c|}
\hline Genetic polymorphisms & Normal controls $(n=338)$ & Cervical neoplasias ${ }^{a}(n=231)$ & ORs (95\% CIs) & $p$ values & AORs $(95 \% \text { CIs) })^{b}$ & Adjusted $p$ values \\
\hline rs975263 & & & & 0.511 & & 0.532 \\
\hline $\mathrm{AA}^{\mathrm{c}}$ & 228 & 158 & 1.00 & & 1.00 & \\
\hline AG & 94 & 67 & $1.03(0.71-1.49)$ & 0.883 & $1.03(0.70-1.52)$ & 0.891 \\
\hline GG & 15 & 6 & $0.58(0.22-1.52)$ & 0.266 & $0.58(0.21-1.55)$ & 0.275 \\
\hline $\mathrm{AA}^{\mathrm{c}}$ & 228 & 158 & 1.00 & 0.852 & 1.00 & \\
\hline AG/GG & 109 & 73 & $0.97(0.68-1.38)$ & & $0.96(0.66-1.40)$ & 0.844 \\
\hline $\mathrm{AA} / \mathrm{AG}^{\mathrm{c}}$ & 322 & 225 & 1.00 & 0.250 & 1.00 & \\
\hline GG & 15 & 6 & $0.57(0.22-1.50)$ & & $0.57(0.21-1.53)$ & 0.265 \\
\hline rs3095007 & & & & 0.682 & & 0.691 \\
\hline$G^{c}$ & 282 & 198 & 1.00 & & 1.00 & \\
\hline GT & 53 & 30 & $0.81(0.50-1.31)$ & 0.382 & $0.81(0.49-1.34)$ & 0.401 \\
\hline TT & 3 & 2 & $0.95(0.16-5.74)$ & 0.955 & $0.81(0.12-5.30)$ & 0.826 \\
\hline $\mathrm{GG}^{\mathrm{c}}$ & 282 & 198 & 1.00 & 0.391 & 1.00 & \\
\hline GT/TT & 56 & 32 & $0.81(0.51-1.30)$ & & $0.81(0.49-1.32)$ & 0.390 \\
\hline GG/GTc & 335 & 228 & 1.00 & 1.000 & 1.00 & \\
\hline TT & 3 & 2 & $0.98(0.16-5.91)$ & & $0.84(0.13-5.46)$ & 0.852 \\
\hline rs4721888 & & & & 0.237 & & 0.169 \\
\hline $\mathrm{GG}^{\mathrm{c}}$ & 186 & 114 & 1.00 & & 1.00 & \\
\hline GC & 126 & 103 & $1.33(0.94-1.89)$ & 0.106 & $1.41(0.98-2.04)$ & 0.064 \\
\hline $\mathrm{CC}$ & 24 & 14 & $0.95(0.47-1.92)$ & 0.890 & $1.03(0.50-2.12)$ & 0.948 \\
\hline $\mathrm{GG}^{\mathrm{c}}$ & 186 & 114 & 1.00 & 0.159 & 1.00 & \\
\hline $\mathrm{GC} / \mathrm{CC}$ & 150 & 117 & $1.27(0.91-1.78)$ & & $1.35(0.95-1.92)$ & 0.093 \\
\hline GG/GCc & 312 & 217 & 1.00 & 0.613 & 1.00 & \\
\hline $\mathrm{CC}$ & 24 & 14 & $0.84(0.42-1.66)$ & & $0.88(0.43-1.79)$ & 0.725 \\
\hline rs3735615 & & & & 0.326 & & \\
\hline $\mathrm{GG}^{\mathrm{c}}$ & 236 & 167 & 1.00 & & 1.00 & 0.366 \\
\hline GC & 89 & 61 & $0.97(0.66-1.42)$ & 0.870 & $0.98(0.66-1.46)$ & 0.929 \\
\hline $\mathrm{CC}$ & 11 & 3 & $0.39(0.11-1.40)$ & 0.148 & $0.39(0.10-1.44)$ & 0.156 \\
\hline$G^{c}$ & 236 & 167 & 1.00 & 0.596 & 1.00 & \\
\hline $\mathrm{GC} / \mathrm{CC}$ & 100 & 64 & $0.90(0.62-1.31)$ & & $0.91(0.62-1.35)$ & 0.647 \\
\hline GG/GCc & 325 & 228 & 1.00 & 0.136 & 1.00 & \\
\hline $\mathrm{CC}$ & 11 & 3 & $0.39(0.11-1.41)$ & & $0.39(0.11-1.44)$ & 0.157 \\
\hline rs1990172 & & & & 0.606 & & 0.602 \\
\hline $\mathrm{CC}^{\mathrm{c}}$ & 245 & 177 & 1.00 & & 1.00 & \\
\hline CA & 82 & 49 & $0.83(0.55-1.24)$ & 0.356 & $0.83(0.54-1.26)$ & 0.372 \\
\hline AA & 9 & 5 & $0.77(0.25-2.33)$ & 0.643 & $0.73(0.23-2.28)$ & 0.588 \\
\hline $\mathrm{CC}^{\mathrm{c}}$ & 245 & 177 & 1.00 & 0.320 & 1.00 & \\
\hline $\mathrm{CA} / \mathrm{AA}$ & 91 & 54 & $0.82(0.56-1.21)$ & & $0.82(0.54-1.22)$ & 0.323 \\
\hline $\mathrm{CC} / \mathrm{CA}^{c}$ & 327 & 226 & 1.00 & 0.698 & 1.00 & \\
\hline $\mathrm{AA}$ & 9 & 5 & $0.80(0.27-2.43)$ & & $0.76(0.25-2.37)$ & 0.640 \\
\hline
\end{tabular}

Relationships of $\mathrm{MACCl}$ genetic variants with clinicopathological characteristics

Patient with cervical cancer exhibiting genotype GG in MACC1 SNP rs975263 tended to exert more risk to have vaginal invasion than those with AA/AG ( $p=0.042$, OR: $8.70,95 \%$ CI: 0.81-433.22; Table 3). There were no correlations of rs975263 with other clinicopathological parameters. Meanwhile, no other MACC1 SNPs were shown to have relationships with a variety of clinicopathological variables (Table 3 ).

\section{Univariate and multivariate analyses for the relationships of $M A C C I$ genetic variants and various clinicopathological characteristics with 5 years survival rate in cervical cancer patients}

In univariate analysis, cervical cancer patients with AG/GG in MACC1 SNP rs975263 had 5 years survival rate $0.89(95 \% \mathrm{CI}=0.79-0.99)$ as compared to those with AA $0.79(95 \% \quad \mathrm{CI}=0.70-0.88)$ with no significantly different 5 years survival rate $(p=0.195$, $\mathrm{HR}=0.49$, 95\% CI=0.16-1.45; Table 4). Meanwhile, cervical cancer patients with GG in MACC1 SNP rs975263 had 5 years survival rate $0.80 \quad(95 \%$ $\mathrm{CI}=0.45-1.00)$ as compared to those with AA/AG 0.82 (95\% CI=0.75-0.90) with no significantly different 5 years survival rate $(p=0.897, \mathrm{HR}=1.14,95 \%$ $\mathrm{CI}=0.15-8.54$; Table 4). Other MACC1 SNPs were also not significantly related to 5 years survival rate (Table 4). However, advanced clinical stage $(p=0.008$; $\mathrm{HR}=3.64,95 \% \mathrm{CI}=1.40-9.47)$, deep stromal invasion $(p=0.009 ; \mathrm{HR}=4.39,95 \% \mathrm{CI}=1.45-13.23)$, larger tumor diameter $(p=0.009 ; \mathrm{HR}=3.83,95 \% \mathrm{CI}=1.39-10.55)$, positive parametrium invasion $(p=0.009$; $\mathrm{HR}=3.42$, 95\% CI=1.36-8.57) and positive pelvic lymph node metastasis $(p<0.001 ; \mathrm{HR}=7.99,95 \% \quad \mathrm{CI}=3.07-20.82)$ could be significantly associated with worse 5 years survival rate for cervical cancer patients (Table 4). Moreover, in multivariate analysis, only positive 
pelvic lymph node metastasis could be significantly

$\mathrm{HR}=9.98,95 \% \mathrm{CI}=2.64-37.77)$ for cervical cancer predictive of worse 5 years survival rate $(p=0.001$;

patients in Taiwan (Table 5).

Table 2. Genetic polymorphism distributions of metastasis-associated in colon cancer-1 gene Taiwanese women with uterine cervical invasive cancer or precancerous lesions and normal controls

\begin{tabular}{|c|c|c|c|c|c|c|c|c|}
\hline Genetic polymorphisms & $\begin{array}{l}\text { Normal controls } \\
(\mathrm{n}=338)\end{array}$ & $\begin{array}{l}\text { Pre-cancerous lesions } \\
(\mathrm{n}=99)\end{array}$ & $\begin{array}{l}\text { Invasive cancer } \\
(\mathrm{n}=132)\end{array}$ & $p$ values & $\begin{array}{l}\text { AORs } \\
(95 \% \text { CIs })^{a}\end{array}$ & Ad. $p$ values $^{a}$ & $\begin{array}{l}\text { AORs } \\
(95 \% \mathrm{CIs})^{\mathrm{b}}\end{array}$ & Ad. $p$ values ${ }^{b}$ \\
\hline \multicolumn{9}{|l|}{ rs975263 } \\
\hline $\mathrm{AA}^{\mathrm{c}}$ & 228 & 70 & 88 & 0.610 & 1.00 & & 1.00 & \\
\hline AG & 94 & 28 & 39 & & $0.97(0.59-1.61)$ & 0.917 & $1.06(0.65-1.73)$ & 0.810 \\
\hline GG & 15 & 1 & 5 & & $0.22(0.03-1.67)$ & 0.141 & $0.97(0.32-2.92)$ & 0.954 \\
\hline $\mathrm{AA}^{\mathrm{c}}$ & 228 & 70 & 88 & 0.795 & 1.00 & & 1.00 & \\
\hline AG/GG & 109 & 29 & 44 & & $0.87(0.53-1.42)$ & 0.574 & $1.05(0.66-1.67)$ & 0.843 \\
\hline $\mathrm{AA} / \mathrm{AG}^{\mathrm{c}}$ & 322 & 98 & 127 & 0.280 & 1.00 & & 1.00 & \\
\hline GG & 15 & 1 & 5 & & $0.22(0.03-1.67)$ & 0.143 & $0.95(0.32-2.84)$ & 0.928 \\
\hline \multicolumn{9}{|l|}{ rs3095007 } \\
\hline$G^{c}$ & 282 & 81 & 117 & 0.327 & 1.00 & & 1.00 & \\
\hline GT & 53 & 17 & 13 & & $1.12(0.61-2.04)$ & 0.716 & $0.51(0.25-1.04)$ & 0.065 \\
\hline TT & 3 & 0 & 2 & & u.a. & u.a. & $1.40(0.18-10.81)$ & 0.747 \\
\hline $\mathrm{GG}^{\mathrm{c}}$ & 282 & 81 & 117 & 0.321 & 1.00 & & 1.00 & \\
\hline GT/TT & 56 & 17 & 15 & & $1.06(0.58-1.92)$ & 0.854 & $0.56(0.29-1.10)$ & 0.093 \\
\hline GG/GTc & 335 & 98 & 130 & 0.477 & 1.00 & & 1.00 & \\
\hline TT & 3 & 0 & 2 & & u.a. & u.a. & $1.52(0.20-11.65)$ & 0.686 \\
\hline \multicolumn{9}{|l|}{ rs4721888 } \\
\hline $\mathrm{GG}^{\mathrm{c}}$ & 186 & 49 & 65 & 0.395 & 1.00 & & 1.00 & \\
\hline GC & 126 & 46 & 57 & & $1.40(0.88-2.22)$ & 0.154 & $1.41(0.89-2.23)$ & 0.147 \\
\hline $\mathrm{CC}$ & 24 & 4 & 10 & & $0.64(0.21-1.94)$ & 0.433 & $1.43(0.61-3.39)$ & 0.412 \\
\hline $\mathrm{GG}^{\mathrm{c}}$ & 186 & 49 & 65 & 0.371 & 1.00 & & 1.00 & \\
\hline $\mathrm{GC} / \mathrm{CC}$ & 150 & 50 & 67 & & $1.28(0.82-2.01)$ & 0.282 & $1.41(0.91-2.19)$ & 0.127 \\
\hline GG/GCc & 312 & 95 & 122 & 0.500 & 1.00 & & 1.00 & \\
\hline $\mathrm{CC}$ & 24 & 4 & 10 & & $0.55(0.19-1.63)$ & 0.284 & $1.24(0.54-2.85)$ & 0.618 \\
\hline \multicolumn{9}{|l|}{ rs3735615 } \\
\hline$G^{c}$ & 236 & 72 & 95 & 0.679 & 1.00 & & 1.00 & \\
\hline GC & 89 & 26 & 35 & & $0.96(0.58-1.60)$ & 0.883 & $0.98(0.59-1.61)$ & 0.926 \\
\hline $\mathrm{CC}$ & 11 & 1 & 2 & & $0.30(0.04-2.33)$ & 0.247 & $0.49(0.10-2.42)$ & 0.382 \\
\hline $\mathrm{GG}^{\mathrm{c}}$ & 236 & 72 & 95 & 0.862 & 1.00 & & 1.00 & \\
\hline $\mathrm{GC} / \mathrm{CC}$ & 100 & 27 & 37 & & $0.89(0.54-1.47)$ & 0.643 & $0.92(0.57-1.50)$ & 0.745 \\
\hline GG/GCc & 325 & 98 & 130 & 0.320 & 1.00 & & 1.00 & \\
\hline $\mathrm{CC}$ & 11 & 1 & 2 & & $0.30(0.04-2.34)$ & 0.250 & $0.49(0.10-2.43)$ & 0.384 \\
\hline \multicolumn{9}{|l|}{ rs1990172 } \\
\hline $\mathrm{CC}^{\mathrm{c}}$ & 245 & 75 & 102 & 0.688 & 1.00 & & 1.00 & \\
\hline $\mathrm{CA}$ & 82 & 23 & 26 & & $0.92(0.54-1.56)$ & 0.752 & $0.72(0.42-1.25)$ & 0.247 \\
\hline AA & 9 & 1 & 4 & & $0.36(0.05-2.88)$ & 0.335 & $1.09(0.30-3.93)$ & 0.899 \\
\hline $\mathrm{CC}^{\mathrm{c}}$ & 245 & 75 & 102 & 0.590 & 1.00 & & 1.00 & \\
\hline $\mathrm{CA} / \mathrm{AA}$ & 91 & 24 & 30 & & $0.86(0.51-1.45)$ & 0.576 & $0.76(0.45-1.28)$ & 0.301 \\
\hline $\mathrm{CC} / \mathrm{CA}^{c}$ & 327 & 98 & 128 & 0.574 & 1.00 & & 1.00 & \\
\hline AA & 9 & 1 & 4 & & $0.37(0.05-2.93)$ & 0.344 & $1.17(0.33-4.20)$ & 0.812 \\
\hline
\end{tabular}

aAdjusted $p$ values and adjusted odds ratios with their 95\% CIs were calculated using multinomial logistic regression models after controlling age between patients with cervical precancerous lesions and control women. bAdjusted $p$ values and adjusted odds ratios with their $95 \%$ CIs were estimated using multinomial logistic regression models after controlling age between patients with cervical invasive cancer and control women. cUsed as a reference for comparison to estimate the odds ratios of other genotypes. AORs, adjusted odds ratios; $95 \%$ CIs, 95\% confidence intervals; Ad. $p$, adjusted $p$; u.a., unavailable.

Table 3. Associations of genotypic distribution of metastasis-associated in colon cancer-1 gene with clinicopathological characteristics of the patients with invasive cancer of uterine cervix

\begin{tabular}{|c|c|c|c|c|}
\hline & rs975263 & & & \\
\hline Variables $^{\mathrm{a}}$ & $\mathrm{AA} / \mathrm{AG}^{\mathrm{b}}$ & GG & $p$ value & ORs (95\% CIs) \\
\hline Clinical stage & & & 1.000 & \\
\hline stage $I^{b}$ & 74 & 3 & & 1.00 \\
\hline$\geq$ stage II & 50 & 2 & & $0.99(0.08-8.94)$ \\
\hline Pathologic type & & & 0.534 & \\
\hline squamous cell carcinomab & 107 & 4 & & 1.00 \\
\hline adenocarcinoma & 17 & 1 & & $1.57(0.03-17.14)$ \\
\hline Cell grading & & & 0.555 & \\
\hline well (grade 1$)^{b}$ & 18 & 1 & & 1.00 \\
\hline moderate \& poor (grades $2 / 3$ ) & 106 & 4 & & $0.68(0.06-35.29)$ \\
\hline Stromal invasion depth & & & 1.000 & \\
\hline$\leq 10 \mathrm{~mm}^{\mathrm{b}}$ & 63 & 2 & & 1.00 \\
\hline$>10 \mathrm{~mm}$ & 56 & 2 & & $1.13(0.08-15.98)$ \\
\hline Tumor diameter ${ }^{b}$ & & & 1.000 & \\
\hline$\leq 4 \mathrm{~cm}$ & 68 & 3 & & 1.00 \\
\hline
\end{tabular}




\begin{tabular}{|c|c|c|c|c|}
\hline & rs975263 & & & \\
\hline Variablesa & $\mathrm{AA} / \mathrm{AG}^{\mathrm{b}}$ & GG & $p$ value & ORs (95\% CIs) \\
\hline$>4 \mathrm{~cm}$ & 56 & 2 & & $0.81(0.07-7.34)$ \\
\hline Parametrium & & & 1.000 & \\
\hline no invasion ${ }^{\mathrm{b}}$ & 83 & 3 & & 1.00 \\
\hline invasion & 44 & 2 & & $1.26(0.10-11.39)$ \\
\hline Vagina & & & 0.042 & \\
\hline no invasion ${ }^{\mathrm{b}}$ & 87 & 1 & & 1.00 \\
\hline invasion & 40 & 4 & & $8.70(0.81-433.22)$ \\
\hline Pelvic lymph node & & & 0.335 & \\
\hline no metastasis ${ }^{\mathrm{b}}$ & 95 & 5 & & 1.00 \\
\hline metastasis & 32 & 0 & & u.a. \\
\hline
\end{tabular}

Statistical analyses: chi-square or Fisher's exact tests. aSome clinicopathological data could not be obtained from the patients with cervical invasive cancer due to incomplete medical charts or records. bAs a reference. ORs, odds ratios; 95\% CIs, 95\% confidence intervals; u.a., unavailable

Table 4. Univariate analysis for the impact of metastasis-associated in colon cancer-1 gene polymorphisms and various clinicopatholgical parameters on the 5 years survival rate

\begin{tabular}{|c|c|c|c|}
\hline \multirow[t]{2}{*}{ Variables } & \multirow[t]{2}{*}{5 years survival rate $\& 95 \% \mathrm{CI}$} & \multicolumn{2}{|c|}{5 years survival hazard } \\
\hline & & $P$ value & $\mathrm{HR}$ and $95 \% \mathrm{CI}^{\mathrm{b}}$ \\
\hline \multicolumn{4}{|c|}{ metastasis-associated in colon cancer-1 gene polymorphisms } \\
\hline AG/GG vs AA ${ }^{a}$ & $0.89(0.79-0.99)$ vs $0.79(0.70-0.88)$ & 0.195 & $0.49(0.16-1.45)$ \\
\hline \multicolumn{4}{|l|}{ rs3095007 } \\
\hline GT/TT vs GGa & $0.83(0.62-1.00)$ vs $0.82(0.74-0.90)$ & 0.761 & $0.80(0.19-3.44)$ \\
\hline $\begin{array}{l}\text { TT vs GG/GTa } \\
\text { rs4721888 }\end{array}$ & $0.50(0.00-1.00)$ vs $0.83(0.76-0.90)$ & 0.254 & $3.23(0.43-24.15)$ \\
\hline GC/CC vs GGa & $0.81(0.71-0.91)$ vs $0.84(0.74-0.93)$ & 0.651 & $1.23(0.51-2.96)$ \\
\hline \multicolumn{4}{|l|}{ rs3735615 } \\
\hline GC/CC vs GGa & $0.90(0.79-1.00)$ vs $0.80(0.71-0.88)$ & 0.193 & $0.44(0.13-1.51)$ \\
\hline CC vs GG/GCa & $0.50(0.00-1.00)$ vs $0.83(0.76-0.90)$ & 0.254 & $3.23(0.43-24.15)$ \\
\hline \multicolumn{4}{|l|}{ rs1990172 } \\
\hline $\mathrm{CA} / \mathrm{AA}$ vs CCa & $0.89(0.76-1.00)$ vs $0.81(0.72-0.89)$ & 0.395 & $0.59(0.17-2.00)$ \\
\hline $\mathrm{AA}$ vs CC/CAa & $0.75(0.33-1.00)$ vs $0.83(0.75-0.90)$ & 0.702 & $1.48(0.20-11.07)$ \\
\hline \multicolumn{4}{|l|}{ Clinical stage } \\
\hline $\begin{array}{l}\geq \text { stage II vs stage } \\
\text { I }^{a}\end{array}$ & $0.70(0.57-0.83)$ vs $0.91(0.84-0.98)$ & 0.008 & $3.64(1.40-9.47)$ \\
\hline $\begin{array}{l}\text { Pathologic type } \\
\text { adenocarcinoma } \\
\text { vs squamous cell } \\
\text { carcinoma }\end{array}$ & $0.68(0.45-0.92)$ vs $0.84(0.77-0.92)$ & 0.129 & $2.19(0.80-6.03)$ \\
\hline $\begin{array}{l}\text { Cell grading } \\
\text { moderate \& poor } \\
\text { (grades } 2 / 3 \text { ) vs } \\
\text { well (grade } 1)^{\mathrm{a}}\end{array}$ & $0.82(0.740-0.90)$ vs $0.82(0.64-1.00)$ & 0.916 & $0.94(0.27-3.20)$ \\
\hline \multicolumn{4}{|c|}{ Stromal invasion depth } \\
\hline $\begin{array}{l}>10 \mathrm{~mm} \text { vs } \leq 10 \\
\mathrm{~mm}^{\mathrm{a}}\end{array}$ & $0.72(0.61-0.84)$ vs $0.93(0.86-1.00)$ & 0.009 & $4.39(1.45-13.23)$ \\
\hline \multicolumn{4}{|l|}{ Tumor diameter } \\
\hline$>4 \mathrm{~cm}$ vs $\leq 4 \mathrm{~cm}^{\mathrm{a}}$ & $0.71(0.59-0.84)$ vs $0.92(0.85-0.99)$ & 0.009 & $3.83(1.39-10.55)$ \\
\hline $\begin{array}{l}\text { Parametrium } \\
\text { invasion vs no } \\
\text { invasion }\end{array}$ & $0.68(0.54-0.83)$ vs $0.90(0.83-0.97)$ & 0.009 & $3.42(1.36-8.57)$ \\
\hline \multicolumn{4}{|l|}{ Vagina } \\
\hline $\begin{array}{l}\text { invasion vs no } \\
\text { invasiona }^{\text {a }}\end{array}$ & $0.75(0.61-0.90)$ vs $0.85(0.77-0.93)$ & 0.240 & $1.70(0.70-4.10)$ \\
\hline \multicolumn{4}{|l|}{ Pelvic lymph node } \\
\hline metastasis vs no & $0.53(0.35-0.71)$ vs $0.93(0.87-0.98)$ & $<0.001$ & $7.99(3.07-20.82)$ \\
\hline
\end{tabular}

Statistical analyses: Kaplan-Meier curve model. aAs a comparison reference. ${ }^{b} \mathrm{HR}$, hazard ratio and $95 \%$ CI, 95\% confidence interval for metastasis-associated in colon cancer-1 genetic variants and clinicopathological variables, compared to their respective controls.

\section{Discussion}

As far as we know, no study investigates the associations of MACC1 SNPs with the development of cervical cancer and patient prognosis in Taiwanese women. Therefore, we conducted this study to explore the involvement of MACC1 SNPs in uterine cervical carcinogenesis. However, we could not find significantly different genotypic frequencies of 5 MACC1 SNPs between patients with cervical neoplasia and normal controls in Taiwanese women. Even after the patients with cervical neoplasias group was classified into those with invasive or pre-cancerous subgroups, and age was controlled, there were still no genotypic distributions among patients with invasive cancer, those with pre-cancerous lesions and normal controls.

Table 5. Multivariate analysis for impact of metastasis-associated in colon cancer-1 gene polymorphisms and various clinicopatholgical parameters on the 5 years survival rate of the patients with uterine cervical cancer

\begin{tabular}{|c|c|c|}
\hline \multirow[b]{2}{*}{ Variables } & \multicolumn{2}{|c|}{5 years survival hazard } \\
\hline & $p$ value & $\mathrm{HR} \& 95 \% \mathrm{CI}^{\mathrm{b}}$ \\
\hline \multicolumn{3}{|c|}{ metastasis-associated in colon cancer- 1 gene polymorphisms } \\
\hline \multicolumn{3}{|l|}{ rs975263 } \\
\hline AG/GG vs $A^{a}$ & 0.763 & $0.59(0.02-17.88)$ \\
\hline GG vs AA/AGa & 0.978 & u.a. \\
\hline \multicolumn{3}{|l|}{ rs3095007 } \\
\hline GT/TT vs GGa & 0.805 & $0.63(0.02-25.69)$ \\
\hline TT vs GG/GTa & 0.933 & u.a. \\
\hline \multicolumn{3}{|l|}{ rs4721888 } \\
\hline GC/CC vs GGa & 0.509 & $1.50(0.45-4.93)$ \\
\hline CC vs GG/GCa & 0.848 & $1.39(0.09-18.67)$ \\
\hline \multicolumn{3}{|l|}{ rs3735615 } \\
\hline GC/CC vs GGa & 0.542 & $0.32(0.01-12.08)$ \\
\hline CC vs GG/GCa & u.a. & u.a. \\
\hline \multicolumn{3}{|l|}{ rs1990172 } \\
\hline $\mathrm{CA} / \mathrm{AA}$ vs $\mathrm{CC}^{\mathrm{a}}$ & 0.653 & $2.43(0.05-116.11$ \\
\hline $\mathrm{AA}$ vs $\mathrm{CC} / \mathrm{CA}^{\mathrm{a}}$ & 0.996 & u.a. \\
\hline \multicolumn{3}{|l|}{ Pelvic lymph node } \\
\hline metastasis vs no metastasisa & 0.001 & $9.98(2.64-37.77)$ \\
\hline
\end{tabular}

MACC1 up-regulation was in the beginning identified to promote tumor proliferation, invasion, and metastasis in colon [1]. High expression of MACC1 was also found in lung [20], breast [21], ovarian [22] and cervical cancers [7]. The genetic polymorphisms may affect the promoter activity and gene expression, therefore SNPs probably display influences on the tumor growth, invasion or 
metastasis such as breast, ovarian and oral cancers [12, 23-32]. The MACC1 SNP rs975263 (S515L), which is a nonsynonymous SNP exchanging serine to leucine, is situated at codon 515 in exon 5. In this study, it was however not found to have an impact on cervical tumorigenesis. This may be in agreement with the finding of Schmid et al. that exchange of leucine with serine in the MACC1 gene exerted no influence on the expression level of MACC1 mRNA in colorectal cancer [18]. The MACC1 SNP rs4721888 (L31V) is in exon 4, leading to leucine exchange to valine [18]. However, the impact on the protein structure is in doubt because leucine and valine pertain to the group of nonpolar amino acids. MACC1 SNPs rs975263 (S515L) and rs4721888 (L31V) have been predicted to be probably benign in characteristic $[33,34]$. MACC1 SNP rs3735615 (R804T) is in exon 7, which exchanges arginine to threonine. But, our study could not demonstrate its association with cervical cancer in Taiwanese women. Meanwhile, both rs1990172 (A29858C) and rs3095009 (C77360T) are not in coding exon but in intron regions, which exhibit no impact on coding exon of MACC1 gene [13, 18]. Moreover, Zheng et al. found no significant difference in the allele or genotype distribution of the MACC1 SNPs between hepatocellular carcinoma (HCC) tissues and adjacent normal tissues, which showed that MACC1 SNPs probably have no influence on the risk of development of HCC [35].

The impact of MACC1 SNPs on the clinicopathological variables of uterine cervical cancer was then examined. Cervical cancer patients with variant homozygote GG in MACC1 rs975263 tended to have vaginal invasion, as compared to those with AA/AG. However, no statistically significant difference was reached. There was no association of rs975263 with other clinicopathological parameters. Furthermore, other MACC1 SNPs displayed no significant relationships with clinicopathological characteristics. Although MACC1 overexpression was previously demonstrated to be associated with colon cancer metastasis [1], Schmid et al. revealed that MACC1 SNPs were not related to clinical parameters such as stage and lymph node invasion in colorectal cancer [18]. However, Dai et al. identified that the distributions of genotype GC and GC/CC in MACC1 SNP rs4721888 were higher in Chinese women with breast cancer, as compared to genotype GG [15]. The genotype GC/CC in rs4721888 was significantly associated with lymph node metastasis.

MCAA1 has been demonstrated to be associated with prognosis of a variety of cancers, such as gastric cancer [36], lung adenocarcinoma [20], pancreatic cancer [37] and rectal cancer [38]. The 5 years survival rate for patients with high MACC1 expression in the primary colorectal cancer has been identified to be only $15 \%$ as compared to $80 \%$ for those with low MACC1 expression [1]. Its high expression was significantly associated with stage, pelvic lymph node metastasis and poor survival in uterine cervical cancer [7]. Moreover, Radhakrishnan et al. concluded that MACC1 regulates tumor cell metastasis and could act as a predictive marker for cancer therapies [39]. Until now, no study investigates the involvement of MACC1 SNPs in the prognosis of cervical cancer patients. Therefore, we related the associations of MACC1 SNPs and clinicopathological characteristics with prognosis of cervical cancer patients, 5 years survival rate. In univariate and multivariate analyses, all the studied MACC1 SNPs rs975263 (S515L), rs4721888 (L31V) and rs3735615 (R804T) as well as rs1990172 (A29858C) and rs3095009 (C77360T) were not identified to be associated with 5 years survival rate in cervical cancer patients in Taiwan. Consistent with our findings, Schmid et al. found that the identification of coding MACC1 SNPs rs4721888 (L31V), rs975263 (S515L) and rs3735615 (R804T) in primary colorectal tumors does not significantly predict patient survival compared to MACC1 expression analysis alone. Only the genotype AG in MACC1 SNP rs975263 could be identified to be correlated with a decreased survival, but restricted in young colon cancer patients in early stage [18]. In contrast, Lang et al. revealed that MACC1 SNPs rs1990172 could be predictable of decreased overall survival in patients with colorectal cancer [13]. However, the SNP rs1990172 is located within an intronic region of the MACC1 gene and does not influence any splice site of a coding exon. Therefore, whether rs1990172 causes the observed effect should be further delineated. Based on the univariate and multivariate analyses in this study, pelvic lymph node status rather than MACC1 SNPs was the only significantly predictive parameter of 5 years survival rate in Taiwanese patients with cervical cancer [40, 41].

The study has some limitations. Firstly, a larger cohort of patients is necessary. Cervical cancer patients presenting genotype GG in MACC1 SNP rs975263 tended to have more risk to develop vaginal invasion than those with AA/AG. But, this did not reach a statistical significance. Therefore, more cases may be needed to explore the role of SNPs rs975263 (S515L) that is in coding exon 5. Secondly, the mechanisms by which SNPs affect the function of MACC1 gene should be further delineated. Thirdly, in addition to coding exon and intron regions, other SNPs that are in the promoter region or the 3'-untranslated region where microRNAs may interact, should be included to investigate. 


\section{Acknowledgments}

This work was supported by grants from Chung Shan Medical University and Chi-Mei Foundation Medical Center (CSMU-CMMC-107-03).

\section{Competing Interests}

The authors have declared that no competing interest exists.

\section{References}

[1] Stein U, Walther W, Arlt F, Schwabe H, Smith J, Fichtner I, et al. MACC1, a newly identified key regulator of HGF-MET signaling, predicts colon cancer metastasis. Nat Med 2009; 15: 59-67.

[2] Chen S, Zong ZH, Wu DD, Sun KX, Liu BL, Zhao Y. The role of metastasis-associated in colon cancer 1 (MACC1) in endometrial carcinoma tumorigenesis and progression. Mol Carcinog 2017; 56: 1361-1371.

[3] Stein U, Burock S, Herrmann P, Wendler I, Niederstrasser M, Wernecke KD, et al. Circulating MACC1 transcripts in colorectal cancer patient plasma predict metastasis and prognosis. PLoS One 2012; 7: e49249.

[4] Stein U, Smith J, Walther W, Arlt F. MACC1 controls Met: what a difference an Sp1 site makes. Cell Cycle 2009; 8: 2467-2469.

[5] Birchmeier C, Birchmeier W, Gherardi E, Vande Woude GF. Met, metastasis, motility and more. Nat Rev Mol Cell Biol 2003; 4: 915-925.

[6] Boccaccio C, Comoglio PM. Invasive growth: a MET-driven genetic programme for cancer and stem cells. Nat Rev Cancer 2006; 6: 637-645.

[7] Zhou X, Xu CJ, Wang JX, Dai T, Ye YP, Cui YM, et al. Metastasis-Associated in Colon Cancer-1 Associates With Poor Prognosis and Promotes Cell Invasion and Angiogenesis in Human Cervical Cancer. Int J Gynecol Cancer 2015; 25: 1353-1363.

[8] Small W, Jr., Bacon MA, Bajaj A, Chuang LT, Fisher BJ, Harkenrider MM, et al. Cervical cancer: A global health crisis. Cancer 2017; 123: 2404-2412.

[9] Martin CM, O'Leary JJ. Histology of cervical intraepithelial neoplasia and the role of biomarkers. Best Pract Res Clin Obstet Gynaecol 2011; 25: 605-615.

[10] Baak JP, Kruse AJ, Robboy SJ, Janssen EA, van Diermen B, Skaland I. Dynamic behavioural interpretation of cervical intraepithelial neoplasia with molecular biomarkers. J Clin Pathol 2006; 59: 1017-1028.

[11] Su SC, Hsieh MJ, Yang WE, Chung WH, Reiter RJ, Yang SF. Cancer metastasis: Mechanisms of inhibition by melatonin. J Pineal Res 2017; 62:

[12] Shastry BS. SNPs: impact on gene function and phenotype. Methods Mol Biol 2009; 578: 3-22.

[13] Lang AH, Geller-Rhomberg S, Winder T, Stark N, Gasser K, Hartmann B, et al. A common variant of the MACC1 gene is significantly associated with overall survival in colorectal cancer patients. BMC Cancer 2012; 12: 20.

[14] Wu HH, Liu YF, Yang SF, Lin WL, Chen SC, Han CP, et al. Association of single-nucleotide polymorphisms of high-mobility group box 1 with susceptibility and clinicopathological characteristics of uterine cervical neoplasia in Taiwanese women. Tumour Biol 2016;

[15] Dai ZJ, Liu XH, Kang HF, Wang XJ, Jin TB, Zhang SQ, et al. Genetic Variation in Metastasis-Associated in Colon Cancer-1 and the Risk of Breast Cancer Among the Chinese Han Population: A STROBE-Compliant Observational Study. Medicine (Baltimore) 2016; 95: e2801.

[16] Horvat M, Potocnik U, Repnik K, Kavalar R, Zadnik V, Potrc S, et al. Single Nucleotide Polymorphisms in Genes MACC1, RAD18, MMP7 and SDF-1a As Prognostic Factors in Resectable Colorectal Cancer. Radiol Oncol 2017; 51: 151-159.

[17] Muendlein A, Hubalek M, Geller-Rhomberg S, Gasser K, Winder T, Drexel H, et al. Significant survival impact of MACC1 polymorphisms in HER2 positive breast cancer patients. Eur J Cancer 2014; 50: 2134-2141.

[18] Schmid F, Burock S, Klockmeier K, Schlag PM, Stein U. SNPs in the coding region of the metastasis-inducing gene MACC1 and clinical outcome in colorectal cancer. Mol Cancer 2012; 11: 49

[19] Su SC, Hsieh MJ, Chou YE, Fan WL, Yeh CB, Yang SF. Effects of RAGE Gene Polymorphisms on the Risk and Progression of Hepatocellular Carcinoma. Medicine (Baltimore) 2015; 94: e1396.

[20] Shimokawa H, Uramoto H, Onitsuka T, Chundong G, Hanagiri T, Oyama T, et al. Overexpression of MACC1 mRNA in lung adenocarcinoma is associated with postoperative recurrence. J Thorac Cardiovasc Surg 2011; 141: 895-898.

[21] Huang Y, Zhang H, Cai J, Fang L, Wu J, Ye C, et al. Overexpression of MACC1 and Its significance in human Breast Cancer Progression. Cell Biosci 2013; 3: 16.

[22] Li H, Zhang H, Zhao S, Shi Y, Yao J, Zhang Y, et al. Overexpression of MACC1 and the association with hepatocyte growth factor/c-Met in epithelial ovarian cancer. Oncol Lett 2015; 9: 1989-1996.

[23] Su CW, Chien MH, Lin CW, Chen MK, Chow JM, Chuang CY, et al. Associations of genetic variations of the endothelial nitric oxide synthase gene and environmental carcinogens with oral cancer susceptibility and development. Nitric Oxide 2018; 79: 1-7.
[24] Yang SF, Liu YF, Cheng CW, Yang WE, Lin WL, Ko JL, et al. Impact of microRNA-34a and polymorphism of its target gene CA9 on susceptibility to uterine cervical cancer. Oncotarget 2017; 8: 77860-77871.

[25] Yarden RI, Friedman E, Metsuyanim S, Olender T, Ben-Asher E, Papa MZ. MDM2 SNP309 accelerates breast and ovarian carcinogenesis in BRCA1 and BRCA2 carriers of Jewish-Ashkenazi descent. Breast Cancer Res Treat 2008; 111: 497-504.

[26] Pan W, Liu L, Wei J, Ge Y, Zhang J, Chen H, et al. A functional IncRNA HOTAIR genetic variant contributes to gastric cancer susceptibility. Mol Carcinog 2016; 55: 90-96.

[27] Su CW, Chen MK, Hung WC, Yang SF, Chuang CY, Lin CW. Functional variant of CHI3L1 gene is associated with neck metastasis in oral cancer. Clin Oral Investig 2019; 23: 2685-2694.

[28] Su SC, Hsieh MJ, Lin CW, Chuang CY, Liu YF, Yeh CM, et al. Impact of HOTAIR Gene Polymorphism and Environmental Risk on Oral Cancer. J Dent Res 2018; 97: 717-724.

[29] Cheng HL, Liu YF, Su CW, Su SC, Chen MK, Yang SF, et al. Functional genetic variant in the Kozak sequence of WW domain-containing oxidoreductase (WWOX) gene is associated with oral cancer risk. Oncotarget 2016; 7: 69384-69396.

[30] Lin CW, Yang WE, Lee WJ, Hua KT, Hsieh FK, Hsiao M, et al. Lipocalin 2 prevents oral cancer metastasis through carbonic anhydrase IX inhibition and is associated with favourable prognosis. Carcinogenesis 2016; 37: 712-722.

[31] Su SC, Hsieh M], Liu YF, Chou YE, Lin CW, Yang SF. ADAMTS14 Gene Polymorphism and Environmental Risk in the Development of Oral Cancer. PLoS One 2016; 11: e0159585.

[32] Yeh CM, Lin CW, Chuang CY, Liu YF, Chou CH, Yang SF, et al. Functional Genetic Variant of Long Pentraxin 3 Gene Is Associated With Clinical Aspects of Oral Cancer in Male Patients. Front Oncol 2019; 9: 581.

[33] Kumar P, Henikoff S, Ng PC. Predicting the effects of coding non-synonymous variants on protein function using the SIFT algorithm. Nat Protoc 2009; 4: 1073-1081.

[34] Adzhubei IA, Schmidt S, Peshkin L, Ramensky VE, Gerasimova A, Bork P, et al. A method and server for predicting damaging missense mutations. Nat Methods 2010; 7: 248-249.

[35] Zheng Z, Gao S, Yang Z, Xie H, Zhang C, Lin B, et al. Single nucleotide polymorphisms in the metastasis-associated in colon cancer-1 gene predict the recurrence of hepatocellular carcinoma after transplantation. Int J Med Sci 2014; 11: 142-150.

[36] Wang L, Wu Y, Lin L, Liu P, Huang H, Liao W, et al. Metastasis-associated in colon cancer-1 upregulation predicts a poor prognosis of gastric cancer, and promotes tumor cell proliferation and invasion. Int J Cancer 2013; 133: 1419-1430.

[37] Wang G, Kang MX, Lu WJ, Chen Y, Zhang B, Wu YL. MACC1: A potential molecule associated with pancreatic cancer metastasis and chemoresistance. Oncol Lett 2012; 4: 783-791.

[38] Kawamura M, Saigusa S, Toiyama Y, Tanaka K, Okugawa Y, Hiro J, et al. Correlation of MACC1 and MET expression in rectal cancer after neoadjuvant chemoradiotherapy. Anticancer Res 2012; 32: 1527-1531.

[39] Radhakrishnan H, Walther W, Zincke F, Kobelt D, Imbastari F, Erdem M, et al. MACC1-the first decade of a key metastasis molecule from gene discovery to clinical translation. Cancer Metastasis Rev 2018; 37: 805-820.

[40] Kamura T, Tsukamoto N, Tsuruchi N, Saito T, Matsuyama T, Akazawa K, et al. Multivariate analysis of the histopathologic prognostic factors of cervical cancer in patients undergoing radical hysterectomy. Cancer 1992; 69: 181-186.

[41] Choi KH, Kim JY, Lee DS, Lee YH, Lee SW, Sung S, et al. Clinical impact of boost irradiation to pelvic lymph node in uterine cervical cancer treated with definitive chemoradiotherapy. Medicine (Baltimore) 2018; 97: e0517. 